\title{
Evaluation of the Effect of Fluctuation of Absolute Value for Diagnostic Accuracy of Fatigue Crack Monitoring Via Statistical Diagnostic Method Using Correlation between Sensors*
}

\author{
Atsushi IWASAKI ${ }^{* *}$, Akihiro MORIMOTO ${ }^{* * *}$, Masataka YATOMI ${ }^{* * * *}$ \\ and Tadashi KIMURA ${ }^{* * * * *}$ \\ **Department of Mechanical Engineering, Gunma University, 1-5-1 Tenjincho, Kiryu city, Gunma, Japan \\ ${ }^{* *}$ Graduate School of Engineering, Gunma University, 1-5-1 Tenjincho, Kiryu city, Gunma, Japan \\ Email: aiwasaki@gunma-u.ac.jp \\ ****IHI Corporation \\ ${ }^{* * * *} \mid \mathrm{HI}$ Inspection \& Instrumentation Co., Ltd
}

\begin{abstract}
This research is about improvement of the diagnostic accuracy of the fatigue crack monitoring via the statistical diagnostic method. Our research group proposes an unsupervised damage diagnostic method named SI-F method which diagnoses the damage from detecting the change of correlation between sensors caused by the initiation or propagation of the damage via the statistical evaluation. By the method, correlation between sensors is identified by using the response surface and the change of them is statistically investigated with the F-test. To identify the crack length by the method, identification about the relation between the crack length and the $F_{0}$ statistic is required. Then in this research, to evaluate effect of the regression error, the noise magnitude and the fluctuation of the external force to the relation, numerical simulation was conducted. For the simulation, two sets of data, one with constant load and one with variable load, are generated and compared. And the applicability of the result of the simulation is experimentally investigated. Finally, the results indicate that the $F_{0}$ affected by the regression error and the noise magnitude but not affected by the external force.
\end{abstract}

Key words: Structural Health Monitoring, Damage Evaluation, Fatigue, Statistical Treatment, Crack Propagation

\section{Introduction}

To prevent the serious failure of civil structures such as bridges and gas pipes, a structural health monitoring system has recently become a technology of interest. Particularly in Japan, it is necessary to minimize maintenance cost, and the development of a system for diagnosing the condition of civil structures in a short time and at low cost is an urgent matter. Then our research group proposed damage diagnostic method, named SI-F method, based on statistical analysis of monitoring data which show high tolerance to the noise and the change of environments of the structure. The method statistically diagnoses structural condition by means of investigating the change of a response surface which identify the relation between multiple sensors. The shape of the response surface implies the correlation between sensors under several environments. If the structure is intact, the correlation between measurements shows no change despite changes in the parameters themselves caused by factors such as noises, changes in the environment, and operating

*Received 16 Nov., 2009 (No. e73) [DOI: 10.1299/jmmp.4.1323]

Copyright $\odot 2010$ by JSME 
vibrations of the structure. Meanwhile, influence of the damage in the structure to the several sensors is not same, thereby causing the correlation between the parameters to change. The method which diagnoses the condition of the structure through the analysis of variations in the correlation between a number of parameters rather than variations in the parameters themselves, thus provides a means of making an accurate automatic diagnosis in an environment that involves constant changes in parameters. Similarity between correlations is tested by F-tests. Damage detection which does not requires learning data of damaged condition of the structures was conducted by following researches of our research $\operatorname{group}^{(1,2)}$.

This paper is about application of the method not to detect the damage but to identification of the damage. To identify the damage, identification about the relation between the damage property and the $F_{0}$ statistic is required. This relation is not affected by the noise added to the sensor measurement. But it is affected by the regression error of the response surface. The regression error is caused by not only inconformity of the regression model but by the fluctuation of magnitude of correlation between sensor caused by the noise magnitude and the fluctuation of the external force act to the structure. To investigate the effect of them to the diagnostic accuracy, numerical simulation and fatigue crack monitoring by the method was conducted in the paper. For the simulation, two simulated sets of data, one with constant load and the other with variable load, are generated and compared. By the experiment, the applicability of the correlation between the test statistic $F_{0}$ and the noise magnitude, the regression model and the regression error caused by them derived from the simulation is examined.

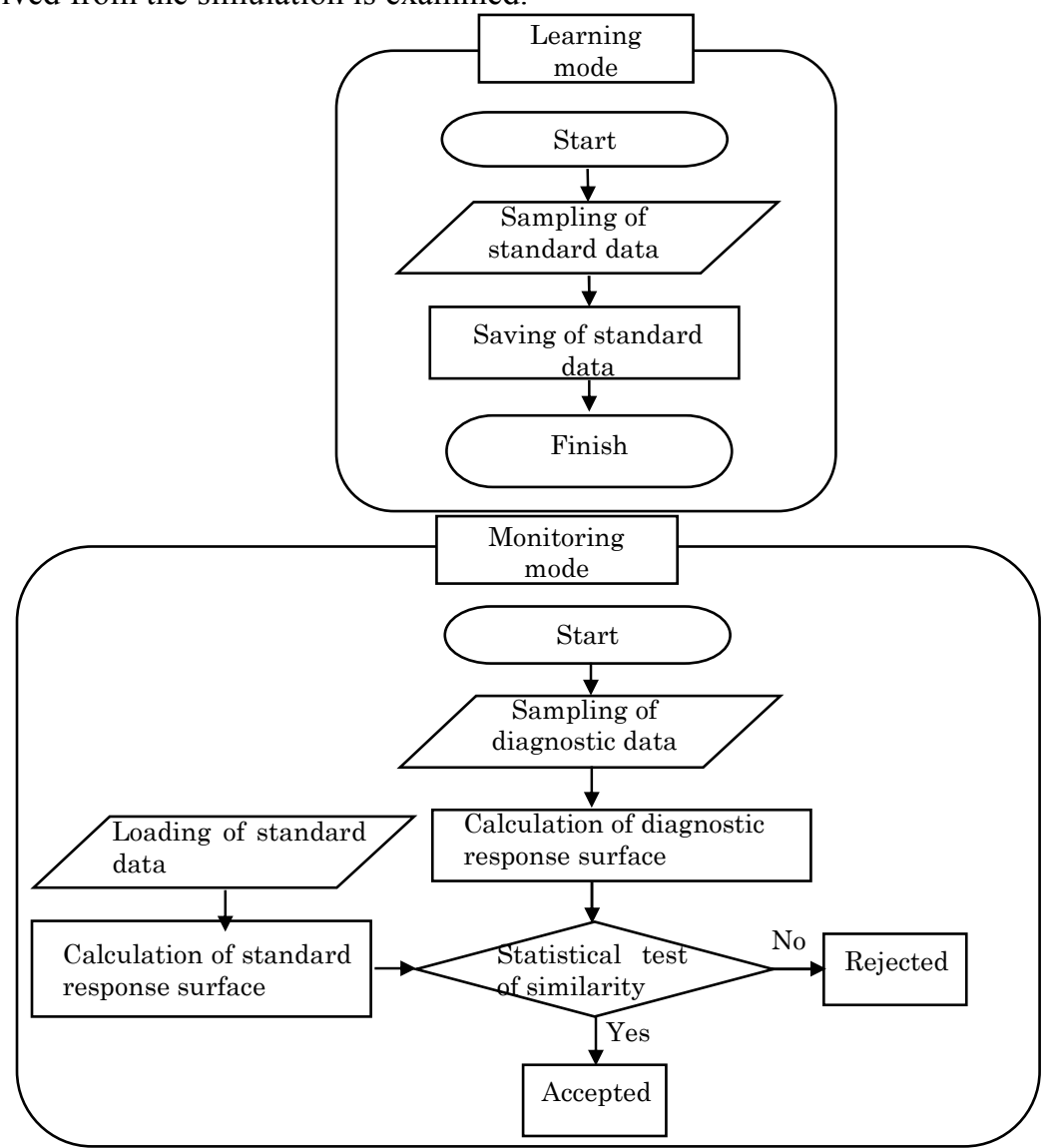

Fig.1 Flow chart of the SI-F method. 


\section{SI-F method}

\subsection{Overview}

In this section, a procedure for the statistical damage diagnosis named the SI-F method (Statistical Structural Integrity Investigation using a statistical F test) is presented. This method consists of a learning mode and a monitoring mode (see Fig.1). The learning mode is a preparation mode and is performed to create the standard benchmarks for the diagnosis. The monitoring mode is a diagnostic mode and is performed to diagnose the structural condition. In the learning mode, reference data are measured from an intact structure. A standard response surface is calculated from the reference data using the response surface method. In the monitoring mode, data from a structure are measured and a diagnostic response surface is calculated. The statistical similarity of the standard response surface and the diagnostic response surface is tested using the $\mathrm{F}$ test for the damage diagnosis. If the response surfaces are estimated to be similar, the conclusion of the diagnosis is that the structure is in an intact condition. Conversely, if the response surfaces are estimated to be dissimilar, the conclusion of the diagnosis is that the structure is damaged.

\subsection{Response surface method}

For the diagnosis, two data sets are prepared. Under the SI-F method, structure damage is detected by detecting changes in the correlation between the sensor outputs. One data set is called the standard data set, and is sampled when the structure is intact. The other data set is called the diagnostic data set, and is sampled when structure is operational. The correlation between the sensor outputs for each data set is identified using the response surface method. The level of similarity between the two data sets is evaluated using the statistical F-test. If the two data sets are estimated to be similar, the structure is reported as intact. Therefore, under this method, damage is detected not from the change in a single sensor output but from the change in the correlation between two or more sensor outputs. We use the response surface method to estimate a relationship between an explained variable $y$ and the explaining variables $x_{i}$ when the exact relationship between the explained variable and the explaining variables is unknown. The response surface is formally expressed as follows.

$$
y=f\left(x_{1}, x_{2} \cdots x_{p}\right)+\varepsilon
$$

Where $p$ is degree of freedom of the response surface. Linear or quadratic polynomials are used to form the general response function. For example, the response surface expressed as a quadratic polynomial is as follows.

$$
y=\beta_{0}+\sum_{i=1}^{k} \beta_{i} x_{i}+\sum_{i=1}^{k} \beta_{i i} x_{i}^{2}+\sum_{i=1}^{k-1} \sum_{j=i+1}^{k} \beta_{i j} x_{i} x_{j}
$$

where $\beta_{i}$ and $\beta_{i j}$ are regression coefficients. Equation (2) can transpose multilinear regression as shown by the next equation by substituting $x_{i} x_{j}=x_{i i}$.

$$
\mathbf{Y}=\mathbf{X} \boldsymbol{\beta}+\boldsymbol{\varepsilon}
$$

The parameter matrices for Eq. (3) are as follows.

$$
\mathbf{Y}=\left\{\begin{array}{c}
y_{1} \\
y_{2} \\
\vdots \\
y_{n}
\end{array}\right\}, \quad \boldsymbol{\beta}=\left\{\begin{array}{c}
\beta_{1} \\
\beta_{2} \\
\vdots \\
\beta_{k}
\end{array}\right\}, \quad \boldsymbol{\varepsilon}=\left\{\begin{array}{c}
\varepsilon_{1} \\
\varepsilon_{2} \\
\vdots \\
\varepsilon_{n}
\end{array}\right\} \mathbf{X}=\left[\begin{array}{cccc}
1 & x_{11} & \cdots & x_{1 k} \\
1 & x_{21} & \cdots & x_{2 k} \\
\vdots & \vdots & \ddots & \vdots \\
1 & x_{n 1} & \cdots & x_{n k}
\end{array}\right]
$$

Where $n$ is number of data and $k$ is number of explaining variables after making the substitution as mentioned above. In this case, the unbiased estimator $\mathbf{b}$ of $\beta$ is calculated by least squares as 


$$
\mathbf{b}=\left(\mathbf{X}^{\mathrm{T}} \mathbf{X}\right)^{-1} \mathbf{X}^{\mathrm{T}} \mathbf{Y}
$$

For the SI-F method, the correlations between the sensor outputs are identified by assuming one of the sensor outputs to be a response, and the other sensor outputs to be the explained variables.

\subsection{Similarity test of the response surface using statistical F test}

Using the proposed method, initiation of damage is diagnosed by detecting changes in the correlations between the sensor outputs, which are identified by the response surface method as discussed above. Changes in the correlations are evaluated by the similarity test of the response surfaces using the statistical $F$ test.

Two response surfaces using the $\mathrm{F}$ test are shown below.

$$
\begin{aligned}
& \mathbf{Y}=\mathbf{X} \boldsymbol{\beta}+\boldsymbol{\varepsilon} \\
& \mathbf{Y}^{\prime}=\mathbf{X}^{\prime} \boldsymbol{\beta}^{\prime}+\boldsymbol{\varepsilon}^{\prime}
\end{aligned}
$$

Index 1 indicates the standard response surface and index 2 indicates the diagnostic response surface. The null hypothesis assumes that the two response surfaces are equal:

$$
\mathbf{H}_{0}: \boldsymbol{\beta}=\boldsymbol{\beta}^{\prime}
$$

$F_{0}$ is defined in next equation, where the error functions $\varepsilon$ and $\varepsilon^{\prime}$ of the two samples are assumed to be independent and identically distributed.

$$
F_{0}=\frac{S S E_{0}-\left(S S E_{1}+S S E_{2}\right)}{S S E_{1}+S S E_{2}} \times \frac{n-2 p}{p}
$$

In this equation, $S S E$ is the square sum of errors of the response surface, $n$ is the total number of data used for a standard and diagnostic response surfaces, and $p$ is the degree of freedom of the response surface. Index 0 indicates all the points of the response surface, index 1 indicates the standard response surface, and index 2 indicates the diagnostic response surface. The all-points response surface is regressed from the two data sets used. This statistical variable $F_{0}$ follows an $\mathrm{F}$ distribution with a first degree of freedom $p$ and a second degree of freedom $n-2 p$. Therefore, if the number of repetitions $r$ and the mean value of $F_{0}$ are calculated, the central limit theorem states that the average of $F_{0}$ will follow the normal distribution of the average $E\left(F_{0}\right)$ with variance $\sigma^{2}\left(F_{0} / r\right)$. The average $E\left(F_{0}\right)$ and variance $\sigma^{2}\left(F_{0} / r\right)$ are shown in the following equation.

$$
\begin{aligned}
& E\left(F_{0}\right)=\frac{n-2 p}{n-2 p-2} \\
& \sigma^{2}\left(F_{0}\right)=\frac{2(n-2 p)^{2}(n-p-2)}{p(n-2 p-2)^{2}(n-2 p-4)}
\end{aligned}
$$

Thus, the equivalence of two response surfaces can be evaluated when the significance level $\alpha$ and the adoption area of equivalence of the response surface null hypothesis are set.

\subsection{Regression accuracy of the response surface}

To evaluate the regression accuracy of the response surface, the adjusted $R$ square $R^{2}$ adj is generally used. This is given by

$$
R^{2}{ }_{a d j}=1-\frac{S S E /(n-p)}{S y /(n-1)}
$$

where $S_{y}$ is the residual sum of squares around the mean value of response $y$. In this study, $S S E_{0}$ is used. 
3. Evaluation of the sensitivity of the damage index for certain damage patterns and noise

\subsection{Numerical data}

In this section, we describe the method of quantifying the relationship between fatigue damage and $F_{0}$ using numerical data. The numerical data simulate different conditions of damage and noise. Standard data use several unit-magnitude sine waves with a frequency of $1 \mathrm{~Hz}$. In this study, considerations are all conducted by three sine waves. Diagnostic data simulate conditions of damage by multiplying standard data by an arbitrary scalar factor. In this study, the scalar factor ranges from 0.01-2. Figs. 1(a) and (b) show the example of the wave form for standard and diagnostic data. The diagnostic data shown is for the case of $\left(y^{\prime}\right.$, $\left.x_{1}{ }^{\prime}, x_{2}{ }^{\prime}\right)=(1.1,1.05,1)$.

Arbitrary noise is added to the standard and diagnostic data to calculate different values of $R_{a d j}^{2}$. The noise is distributed according to a normal distribution, with a mean of 0 and a variance of $\sigma^{2}$. The $\mathrm{S} / \mathrm{N}$ ratio is defined for the range of $1-20$, escalate 0.5 , and the variance is defined to be the target $\mathrm{S} / \mathrm{N}$ ratio. Figs. 2(a)-(d) show examples of wave forms with added noise for $\left(y^{\prime}, x_{1}{ }^{\prime}, x_{2}\right)=(1.1,1.05,1)$. The $\mathrm{S} / \mathrm{N}$ ratios are $20,10,5$, and 1 .

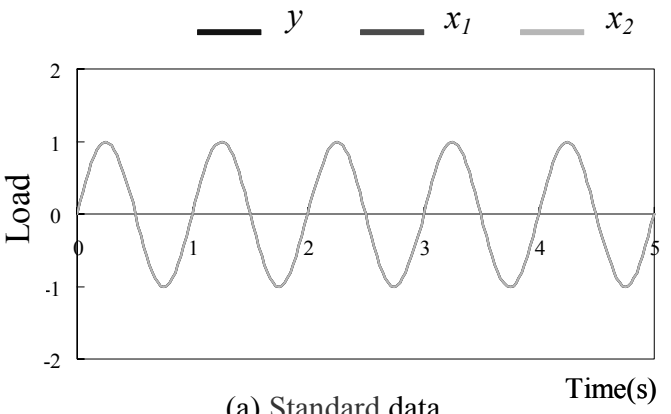

(a) Standard data

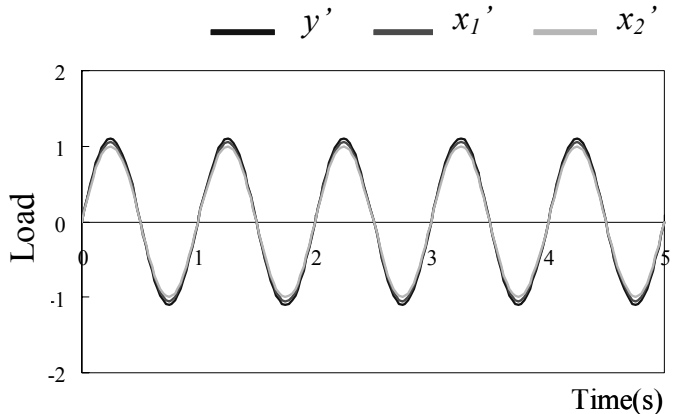

(b) Diagnosis data

Fig.1 Example of wave shape of standard and diagnostic data

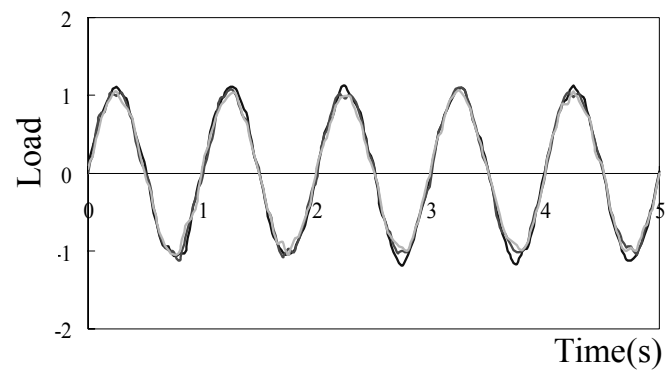

(a) $\mathrm{S} / \mathrm{N}=20$

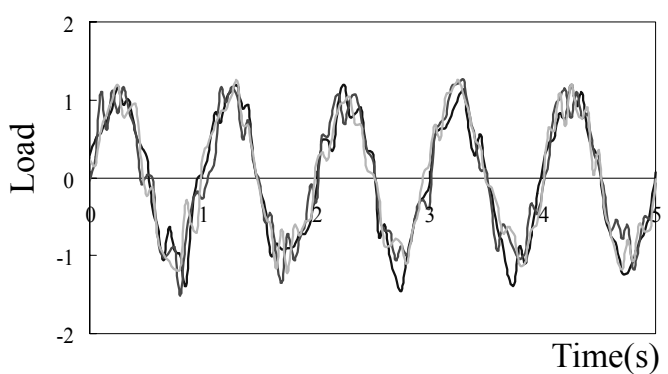

(c) $\mathrm{S} / \mathrm{N}=5$

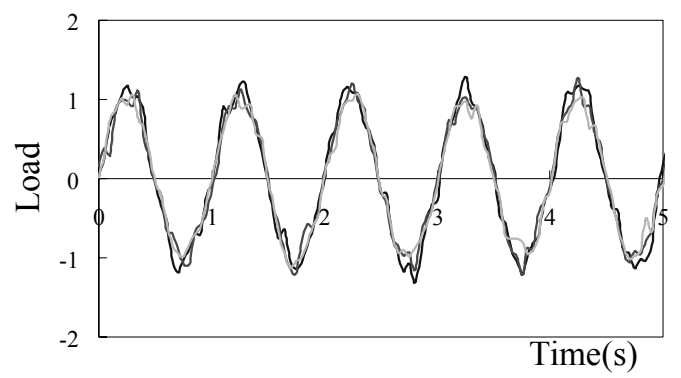

(b) $\mathrm{S} / \mathrm{N}=10$

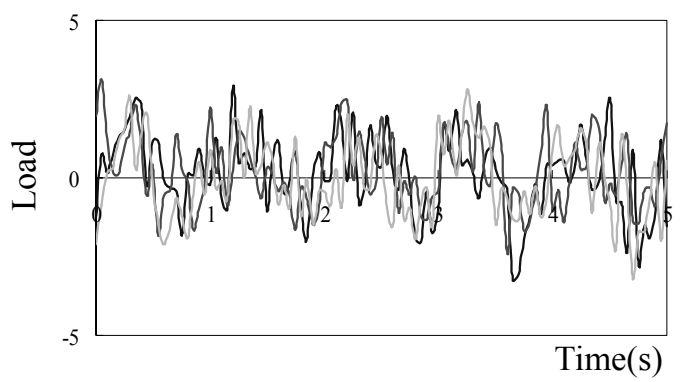

(d) $\mathrm{S} / \mathrm{N}=1$

Fig.2 Examples of wave shape adding noise of $\left(y^{\prime}, x_{1}{ }^{\prime}, x_{2}{ }^{\prime}\right)=(1.1,1.05,1)$ 


\subsection{Calculation of analytical average of $\boldsymbol{F}_{0}$}

Using the numerical data, we calculate the average $F_{0}$ and $R_{a d j}^{2}$ to use in the SI-F method. The analysis conditions are given in Table 1 . In the present study, the $\mathrm{S} / \mathrm{N}$ ratio range is 1-20 and changes escalate 0.5 ; therefore $F_{0}$ and $R_{a d j}^{2}$ are calculated 39 times for the same damage conditions.

Figure 3 shows the relationship between the analytical average of $F_{0}\left(\overline{F_{0}}\right)$ and $R_{a d j}^{2}$ for $\left(y^{\prime}, x_{1}{ }^{\prime}, x_{2}\right)=(1.1,1.05,1)$. The plot allows us to quantify the relationship between arbitrary fatigue damage and $F_{0}$ and adjusted $R_{a d j}^{2}$.

Table 1 Analysis conditions.

\begin{tabular}{|c|c|}
\hline Number of data & 10000 \\
\hline Number of data extracted & 100 \\
\hline Number of times $F_{0}$ is calculated & 100 \\
\hline
\end{tabular}

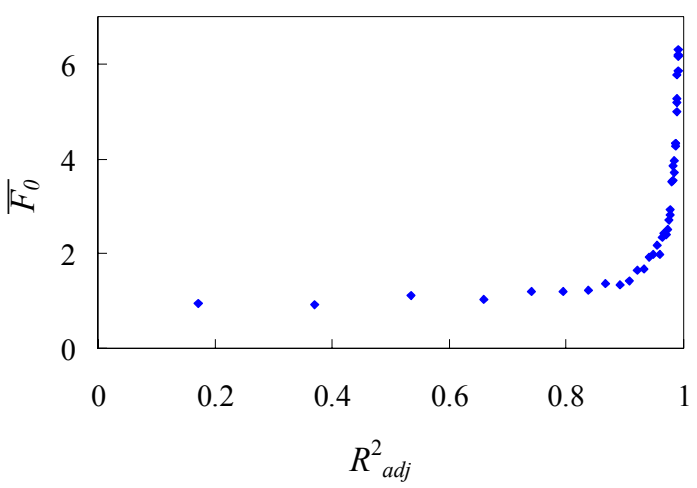

Fig.3 The relationship between $\overline{F_{0}}$ and $R_{a d j}^{2}$ at $\left(y^{\prime}, x_{1}{ }^{\prime}, x_{2}{ }^{\prime}\right)=(1.1,1.05,1)$

\subsection{Evaluation of the sensitivity of the damage index for certain damage patterns and} noise

In this section, we evaluate changes in the correlations between multiple sensors and the strength of the correlations for identifying fatigue damage. This enables us to qualitatively evaluate the relationship between $\overline{F_{0}}$ and $R_{a d j}^{2}$ for arbitrary fatigue damage.

As previously explained the SI-F method identifies changes in the correlations between multiple sensors. This method does not evaluate changes in the modulus of a single sensor output, but rather differences in the variation ratio of each sensor. This is one of the merits of the SI-F method because it does not give a false positive if it detects similar changes in the variation ratio of sensors due to changes in the load and noise conditions for an undamaged structure. Therefore, in this section we evaluate the relation between the fluctuation of $F_{0}$, means sensitivity of the method for the damage, and regression accuracy of the response surface for the types of the change of sensors relation.

Grouping the types of the change of sensor relation follows Fig.4. The combination of change of each sensor outputs affects the change of the response surface is six when the magnitudes of the change of each sensor are assumed large (L) and small (S). And more, the combination of the change of the correlation of each sensor is three when the modulus of remainder of the magnitude of each sensor outputs is used the category of grouping. Therefore, the types of the change of sensors relation are grouped three as follows.

1) The case of the change of the correlation between the variables are small

2) The case of the change of the correlation between explained variable and explaining variables are large

3) The case of the change of the correlation between one explaining variable and the other variables are large

Fig.5 (a)-(d) show plots of the analytical average of $\overline{F_{0}}$ versus $R_{a d j}^{2}$ for the case of the change of the correlation between the variables are small. For the case of Fig.5(a), where 
the changes in the sensor output module are small and no change exists in the correlations between the sensors, $\overline{F_{0}}$ ranges in the vicinity of unity, which is the mean of the distribution of $F_{0}$ (the case for conditions of no damage). Although the case where there is no change in the correlations between the sensors but changes exist in the sensor output moduli escalate much like Fig.5(b), the tendency of smaller $\overline{F_{0}}$ transfers is shown. As a result, it is considered from eq. (8) that as $F_{0}$ varies, $S S E_{2}$ fluctuates more than $S S E_{0}$. For example, $\overline{F_{0}}$ ranges in the vicinity of 0.7 for the case of Fig.5(b); here $\overline{F_{0}}$ is the rejection area, therefore indicating that damage is identified even for the case where there is no change in the sensor correlations but changes exist in the sensor output moduli that highly affects the damage of the structure.

Fig.5(c) and (d) show the case where there are more changes in the correlation of each sensor than those for Fig.5(a) and Fig.5 (b). It is known that $\overline{F_{0}}$ increases significantly with the increase in $R_{a d j}^{2}$, and the configuration of distribution of $\overline{F_{0}}$ transform to unilateral of hyperbolic curve. Asymptotic lines are $R_{a d j}^{2}=1$ and $F_{0}=1$ as shown in Fig.5(c) and (d)

Next, Fig.6(a)-(d) show plots of the relationship between analytical average of $\overline{F_{0}}$ and $R_{a d j}^{2}$ for The case of the change of the correlation between explained variable and explaining variables are large. It is known that $\overline{F_{0}}$ increases more significantly than for changes in the sensor correlations, which are relatively small. And the value of $R_{a d j}^{2}$, one asymptotic line of hyperbolic curve, decreases with changes in the correlation of explained variable $y$, and the other explaining variable increases. These factors indicate that $S S E_{0}$ is larger than in the case of changes in the sensor correlations, which are relatively small in Eq. (11).

Fig.7(a)-(d) show plots of the relationship between analytical average $\overline{F_{0}}$ and $R_{a d j}^{2}$ for the case of the change of the correlation between one explaining variable and the other variables are large. It is known that the configuration of the distribution of $\overline{F_{0}}$ transform to graduated straight line from hyperbolic curve. As a result, it can be considered that one of the sensor of explaining variable, the case where changes in the correlation with explained variable $y$ is larger than the other sensor of explaining variable, contributes to the regression of the response surface more than the other explaining variable, and the tendency of regression of response surface only explained variable and the stronger explaining variable is reflected.

Consequently, the effective of the change in the correlation of multiple sensors and the strength of the correlation for identifying fatigue damage to qualitatively evaluate the relationship between $\overline{F_{0}}$ and $R_{a d j}^{2}$ at arbitrary fatigue damage, the stage of changes in the correlation of each sensor is small. The configuration of distribution of $\overline{F_{0}}$ is a horizontal line range in the vicinity unity. From there, the change of modulus of each sensor output increases and the horizontal line range decreases. When transforming to the stage where the changes in the correlation of each sensor are large from that where the changes are small, the configuration of distribution of $\overline{F_{0}}$ graduated transform to hyperbolic curve, asymptotic lines are $R_{a d j}^{2}=1$ and $F_{0}=1$. From there, the case where change in the correlation of the sensor using the explained variable y increases compared to other sensors using explaining variables, $\overline{F_{0}}$ increases more clearly and gradually decreases the value of $R_{\text {adj }}^{2}$ to being one asymptotic line of the hyperbolic curve. When transforming to the stage where changes in the correlation of all sensors are large from that where the changes are small, the configuration of distribution of $\overline{F_{0}}$ graduated transform to straight line from hyperbolic curve.

As the conclusions of this section, even if the change of correlation is small, $\overline{F_{0}}$ increases high sensitivity as $R_{\text {adj }}^{2}$ increase. As increasing of fluctuation of sensor output by

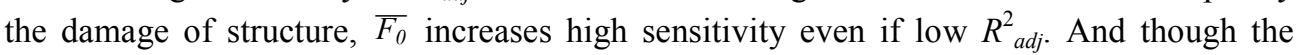
sensitivity decreases by the change of correlation between the each explaining variable, changing the combination of each variables enables higher sensitivity diagnosis, that is 
shown Fig.6(b) and Fig.7(b).

Change of the sensor outputs

Change of the correlation between the sensors

\begin{tabular}{|c|c|c|}
\hline$y^{\prime}$ & $x_{1}{ }^{\prime}$ & $x_{2}{ }^{\prime}$ \\
\hline $\mathbf{L}$ & $\mathbf{L}$ & $\mathbf{L}$ \\
\hline $\mathbf{S}$ & $\mathbf{S}$ & $\mathbf{S}$ \\
\hline $\mathbf{L}$ & $\mathbf{S}$ & $\mathbf{S}$ \\
\hline $\mathbf{S}$ & $\mathbf{L}$ & $\mathbf{L}$ \\
\hline $\mathbf{L}$ & $\mathbf{L}$ & $\mathbf{S}$ \\
\hline $\mathbf{S}$ & $\mathbf{S}$ & $\mathbf{L}$ \\
\hline
\end{tabular}

\begin{tabular}{|c|c|c|c|}
\hline$\left|y^{\prime}-x_{1}{ }^{\prime}\right|$ & $\left|y^{\prime}-x_{2}{ }^{\prime}\right|$ & $\left|x_{1}{ }^{\prime}-x_{2}{ }^{\prime}\right|$ & Group \\
\hline $\mathbf{S}$ & $\mathbf{S}$ & $\mathbf{S}$ & \multirow{2}{*}{$1)$} \\
\cline { 1 - 3 } $\mathbf{S}$ & $\mathbf{S}$ & $\mathbf{S}$ & \\
\hline $\mathbf{L}$ & $\mathbf{L}$ & $\mathbf{S}$ & \multirow{2}{*}{$2)$} \\
\cline { 1 - 3 } $\mathbf{L}$ & $\mathbf{L}$ & $\mathbf{S}$ & \\
\cline { 1 - 3 } $\mathbf{S}$ & $\mathbf{L}$ & $\mathbf{L}$ & \multirow{2}{*}{$3)$} \\
\cline { 1 - 3 } $\mathbf{S}$ & $\mathbf{L}$ & $\mathbf{L}$ & \\
\hline
\end{tabular}

Fig.4 Grouping the types of the change of sensor relation

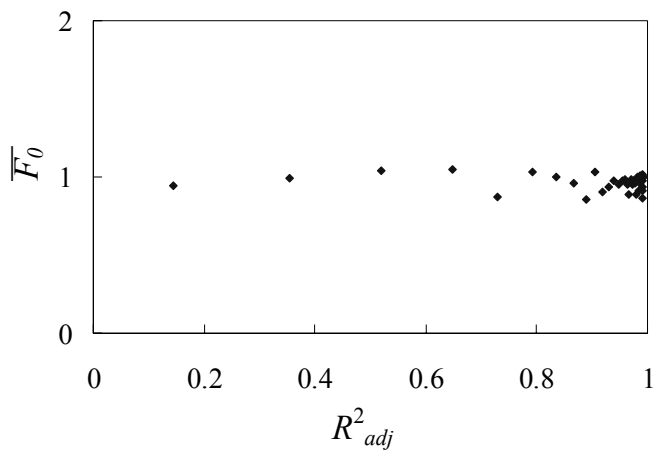

(a) $\left(y^{\prime}, x_{1}{ }^{\prime}, x_{2}\right)=(1.01,1.01,1.01)$

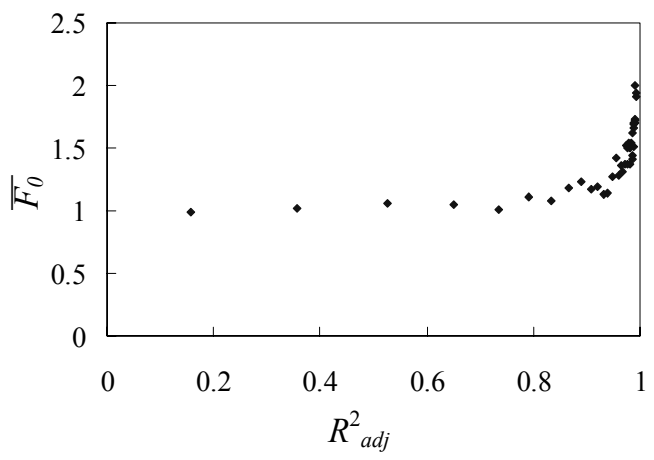

(c) $\left(y^{\prime}, x_{1}{ }^{\prime}, x_{2}{ }^{\prime}\right)=(1.05,1.02,1.01)$

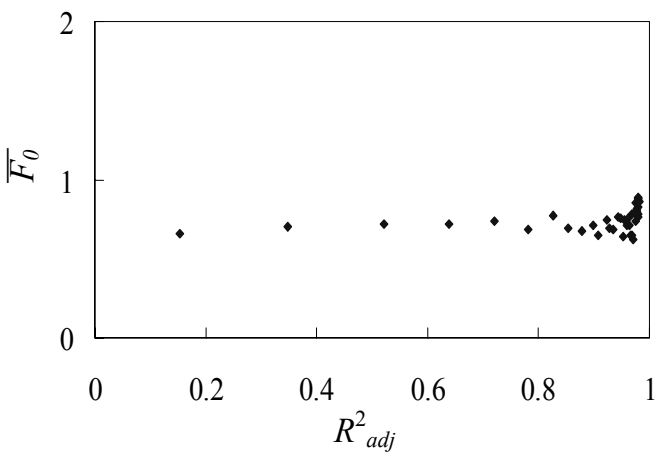

(b) $\left(y^{\prime}, x_{1}{ }^{\prime}, x_{2}{ }^{\prime}\right)=(2.00,1.98,1.98)$

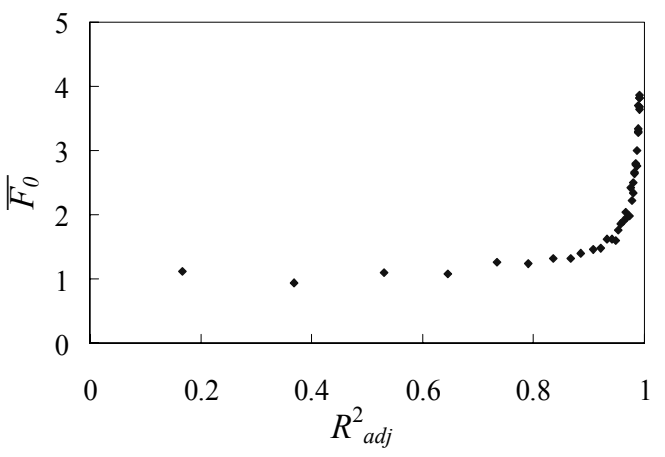

(d) $\left(y^{\prime}, x_{1}{ }^{\prime}, x_{2}{ }^{\prime}\right)=(1.10,1.05,1.02)$

Fig.5 The relationship between analytical $\overline{F_{0}}$ and $R_{\text {adj }}^{2}$ for the case where changes in the correlation of each sensor are relatively small 


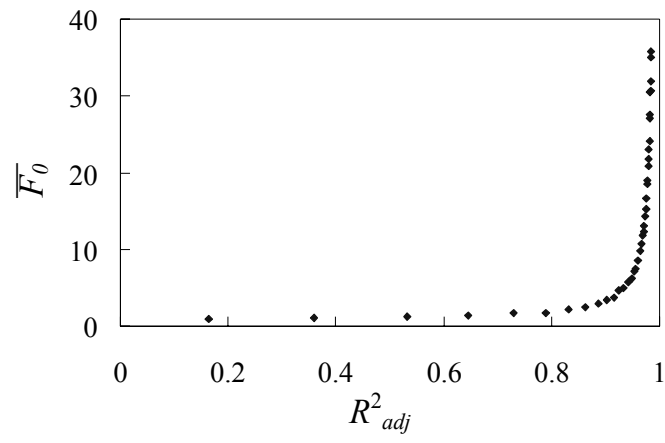

(a) $\left(y^{\prime}, x_{1}{ }^{\prime}, x_{2}\right)=(1.50,1.00,1.00)$

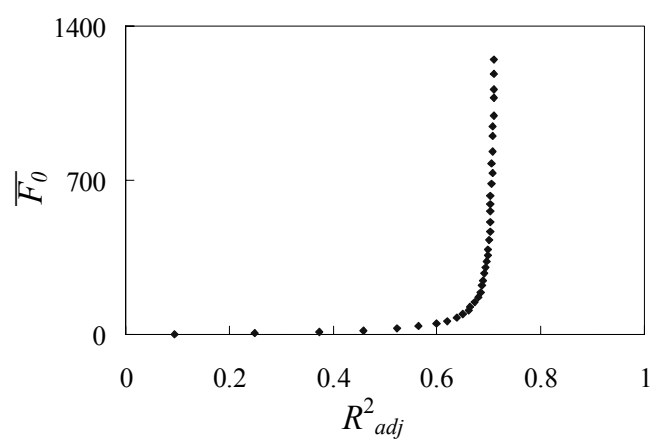

(c) $\left(y^{\prime}, x_{1}{ }^{\prime}, x_{2}{ }^{\prime}\right)=(2.00,0.6,0.6)$

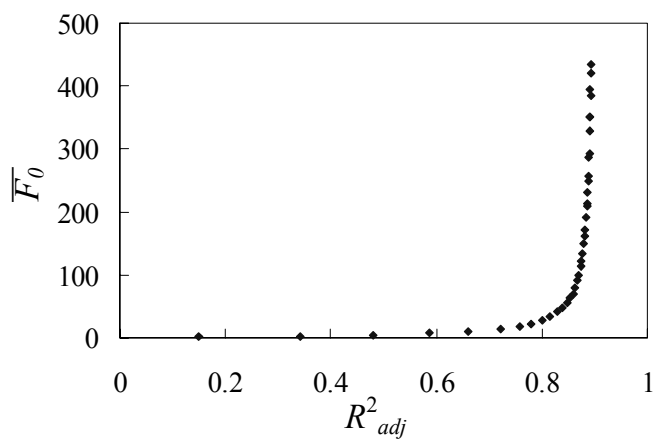

(b) $\left(y^{\prime}, x_{1}{ }^{\prime}, x_{2}\right)=(2.00,1.00,1.00)$

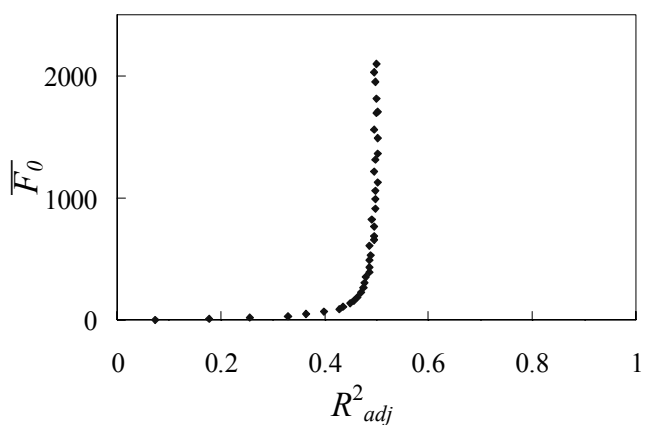

(d) $\left(y^{\prime}, x_{1}{ }^{\prime}, x_{2}{ }^{\prime}\right)=(0.10,1.10,1.10)$

Fig.6 The relationship between analytical $\overline{F_{0}}$ and $R_{\text {adj }}^{2}$ for the case where changes in the correlation of explained variable $y$ are relatively larger than the for other explaining variable

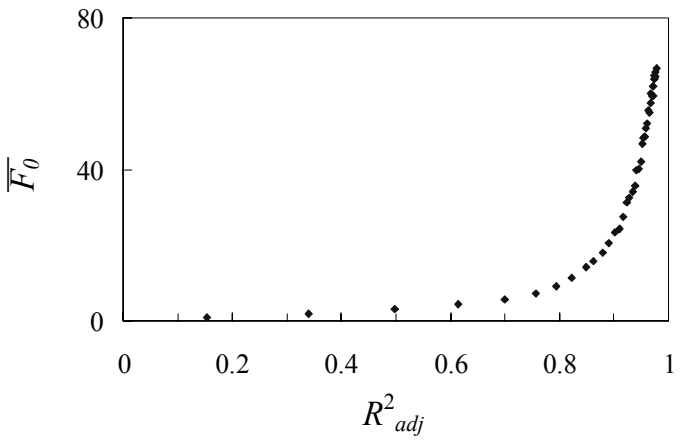

(a) $\left(y^{\prime}, x_{1}{ }^{\prime}, x_{2}{ }^{\prime}\right)=\left(2 \_1.4 \_1\right)$

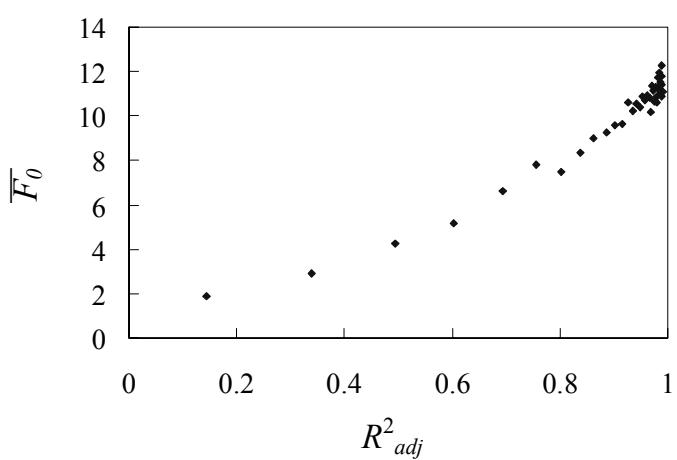

(c) $\left(y^{\prime}, x_{1}{ }^{\prime}, x_{2}{ }^{\prime}\right)=(2,2,0.5)$

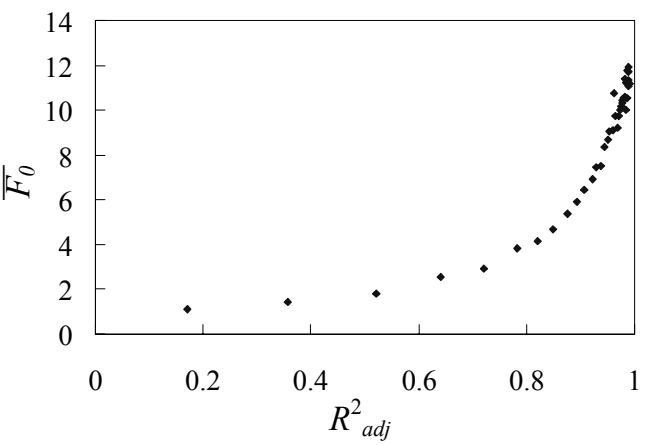

(b) $\left(y^{\prime}, x_{1}{ }^{\prime}, x_{2}{ }^{\prime}\right)=(1,1,2)$

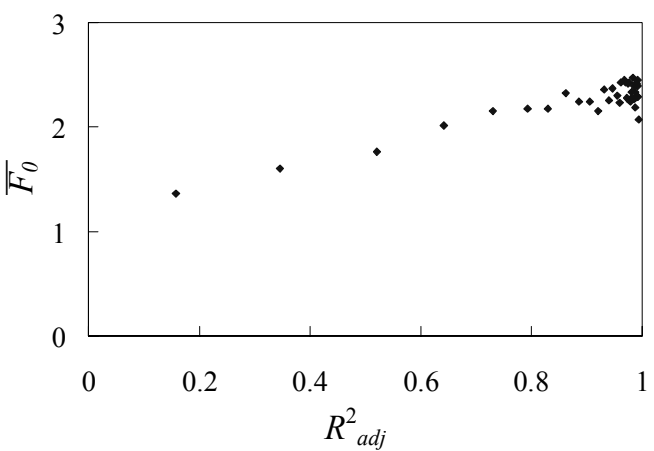

(d) $\left.\left(y^{\prime}, x_{1}{ }^{\prime}, x_{2}{ }^{\prime}\right)=0.8,1.2,0.4\right)$

Fig.7 The relationship between analytical average $\overline{F_{0}}$ and $R_{\text {adj }}^{2}$ for the case where changes in the correlation of each sensor are large 


\section{Evaluating the effect of magnitude of fluctuation}

\subsection{Numerical data for variable load}

The load which act $\mathrm{s}$ on the actual structures is not always constant amplitude load. For example, to a bridge, because of the influence of the traffic condition or the environmental condition and so on, variable amplitude load is worked. In the case of constant amplitude load, the correlation intensity between sensors is constant for every cycle, but in the case of fluctuating load, the peaks differ for every cycle, and the correlation intense differ also at the same noise magnitude. Therefore, the evaluation of the effect of magnitude of fluctuation of the load for diagnostic accuracy is necessary. This section evaluates the effect of magnitude of fluctuation for diagnostic accuracy of fatigue crack monitoring for using numerical data is conducted. And comparison of the relation between average of versus $R_{a d j}^{2}$ of this condition and that of constant amplitude load is conducted.

$-{ }^{y}-x_{1}$

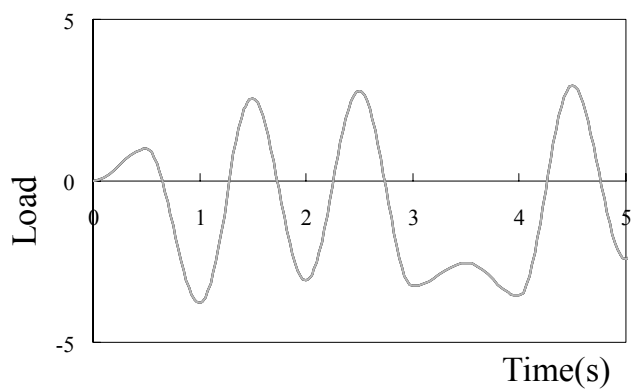

Fig.8 Example of wave shape of standard data

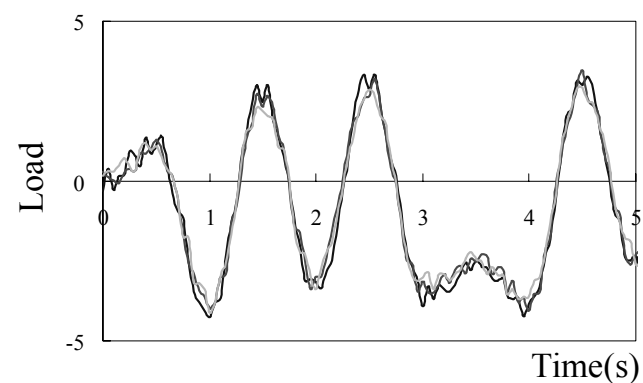

(a) $\mathrm{S} / \mathrm{N}=8$

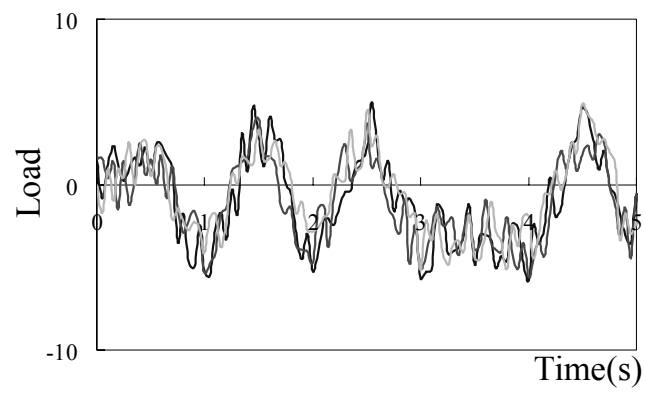

(c) $\mathrm{S} / \mathrm{N}=2$

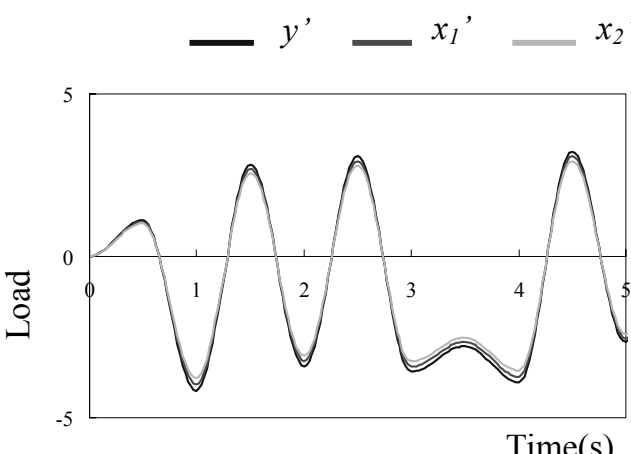

Fig.9 Example of wave shape of diagnostic data

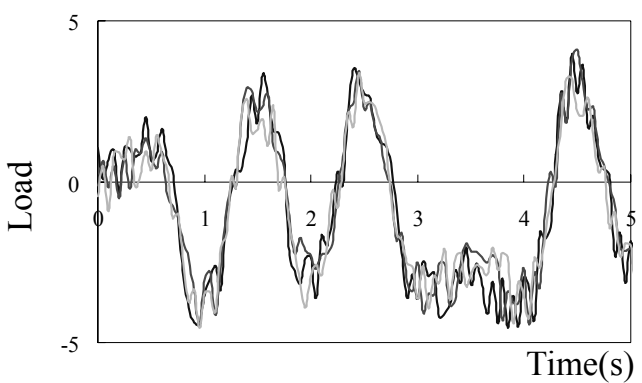

(b) $\mathrm{S} / \mathrm{N}=4$

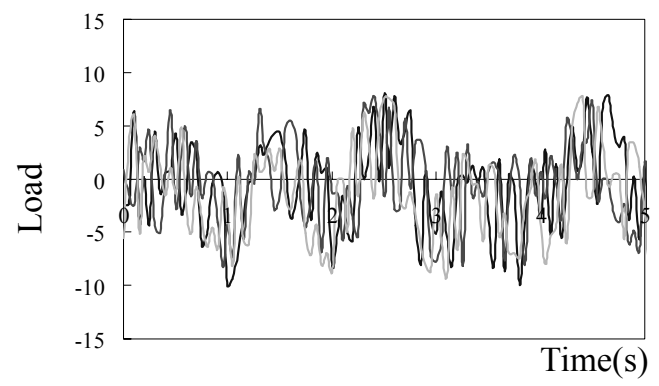

(d) $\mathrm{S} / \mathrm{N}=0.6$

Fig.10 Examples of wave shape adding noise of $\left(y^{\prime}, x_{1}{ }^{\prime}, x_{2}{ }^{\prime}\right)=(1.1,1.05,1)$

\subsection{Generating numerical data to simulate variable loads}

The method for generating numerical data to simulate variable loads is essentially the same as discussed in Section 3.1, except that the wave form is for variable loads. Standard data use three-wave simulated variable loads. The variable load interpolates the peak values sampled randomly from normally distributed data. The mean of the variable load is zero and 
the variance is unity. Fig.8 shows an example of the wave form for standard data. Diagnostic data that simulates a damage condition is obtained by scaling the standard data by an arbitrary scalar factor. In the present study, the scalar range is 0.01-2. Fig.9 shows an example of wave form for diagnostic data for $\left(y^{\prime}, x_{1}{ }^{\prime}, x_{2}\right)=(1.1,1.05,1)$.

The noise distribution follows a normal distribution; the mean is zero and the variance is $\sigma^{2}$. The $\mathrm{S} / \mathrm{N}$ ratio is defined for the range of $0.2-8$, escalate 0.2 , and variance is defined to be the target $\mathrm{S} / \mathrm{N}$ ratio. Fig.10(a)-(d) show examples of wave forms adding noise at $\left(y^{\prime}, x_{1}{ }^{\prime}\right.$, $\left.x_{2}{ }^{\prime}\right)=(1.1,1.05,1)$. The $\mathrm{S} / \mathrm{N}$ ratios are $8,4,2$, and 0.5 .

Using the numerical data, we calculate the average $F_{0}$ and $R_{a d j}^{2}$ to perform the SI-F method. The analysis conditions are the same as given in Table 1 .

\subsection{Comparison with the result of constant load}

Using the numerical data, we calculate the average $F_{0}$ and $R_{a d j}^{2}$ to use in the SI-F method and compare the results with those of the constant load situation. The analysis conditions are the same as given in Table 1. In the present study, $\mathrm{S} / \mathrm{N}$ range is $0.2-8$ and changes escalate 0.2 , so $F_{0}$ and $R_{\text {adj }}^{2}$ are calculation 39 times for the same damage conditions.

Fig.11(a)-(c) show plots of $\overline{F_{0}}$ versus $R_{\text {adj }}^{2}$ for constant load and variable load at $\left(y^{\prime}\right.$, $\left.x_{1}{ }^{\prime}, x_{2}{ }^{\prime}\right)=(1.1,1.05,1),\left(y^{\prime}, x_{1}{ }^{\prime}, x_{2}{ }^{\prime}\right)=(2,0.6,0.6)$, and $\left(y^{\prime}, x_{1}{ }^{\prime}, x_{2}{ }^{\prime}\right)=(0.2,1.6,0.8)$. Every plot nearly conforms to the result of constant load and variable load. The results indicate that $F_{0}$ varies only with the noise magnitude but is not affected by external forces.

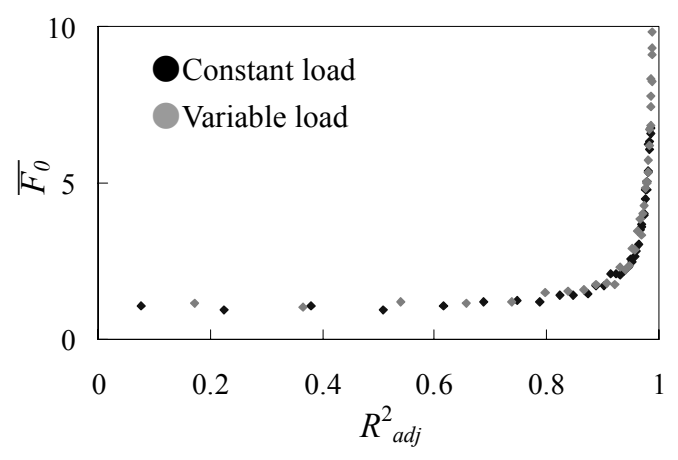

(a) $\left(y^{\prime}, x_{1}^{\prime}, x_{2}\right)=(1.1,1.05,1)$

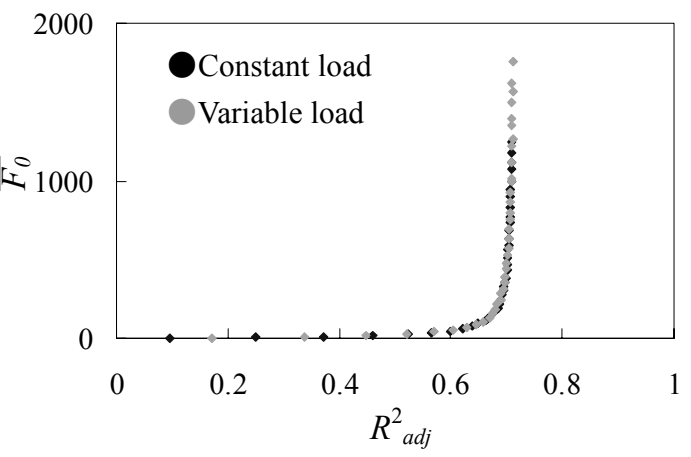

(b) $\left(y^{\prime}, x_{1}{ }^{\prime}, x_{2}{ }^{\prime}\right)=(2,0.6,0.6)$

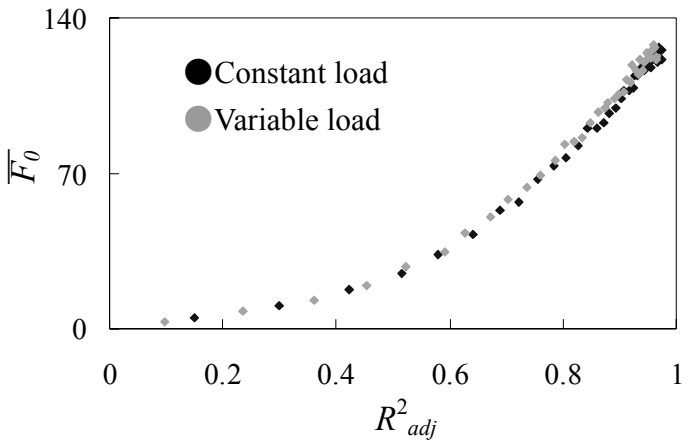

(c) $\left(y^{\prime}, x_{1}{ }^{\prime}, x_{2}{ }^{\prime}\right)=(0.2,1.6,0.8)$

Fig.11 The relationship between $\mathrm{F}_{0 \mathrm{~A}}$ and $\mathrm{R}_{\text {adj }}^{2}$ of constant load and variable load 
5. Comparison of the relation between parameters calculated from the simulation and experiment

This section evaluates the agreement between $F_{0}$, calculated by the experimental data, and the $\overline{F_{0}}$. We investigate whether or not $\overline{F_{0}}$ is applicable as a fatigue index to monitor damage of real structures.

We perform fatigue tests using a mock test structure. Fig.12 shows images of test structures with a simulated L-shaped foot bridge. The test structure material is SS400, and it mainly consists of a beam flange, a column flange, a diaphragm, and a column web. In addition, brackets and base plates are welded to the test structure. The base plate is bolted to the floor. Load fatigue tests bring by fatigue load generator bolted beam upper flange. In the present study, variable load brings test structure. The load frequency is $1 \mathrm{~Hz}$, average of load is $-150 \mathrm{kN}$, amplitude of load is $\pm 150 \mathrm{kN}$.

Crack confirmation is conducted every 75,000 cycles and we use three strain gauges to measure strain with a sampling frequency of $20 \mathrm{~Hz}$. Fig. 13 shows images of set strain gauges and the location of cracks. A crack is found visually at the corner of the test structure at 33,000 cycles. After about 116,500 cycles, the test is completed and the crack has grown to $20 \mathrm{~mm}$. Fig. 14 shows an image of the growth of the fatigue crack.

Next, we evaluate the agreement between $F_{0}$, calculated by experimental data, and $\bar{F}_{0}$. Fig. 15 shows different magnitudes from ch 1, 2, and 3 normalized the standard value for each cycle. Fig. 16 shows $R_{a d j}^{2}$ for each cycle. The conditions under which the experimental data was calculated for the SI-F method are as follows: the explained variable is strain gauge number 1, the explaining variables are strain gauges number 2 and 3 , the number of standard data and diagnostic data is each 10000 , data extracted to calculate $F_{0}$ is 100 , and the number of times $F_{0}$ calculated is $100 . \overline{F_{0}}$ is calculated using numerical data based on Fig.15 and Fig. 16.

In addition, Fig. 17 shows $F_{0}$ and $F_{0}$ with above at each cycle; both are nearly equal. This shows that $\overline{F_{0}}$ can be used as a fatigue index for damage monitoring of real structures.

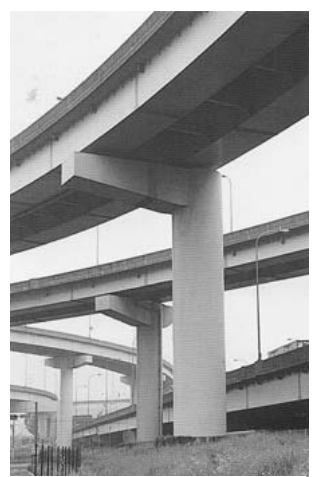

http://smartshimbun.jp/

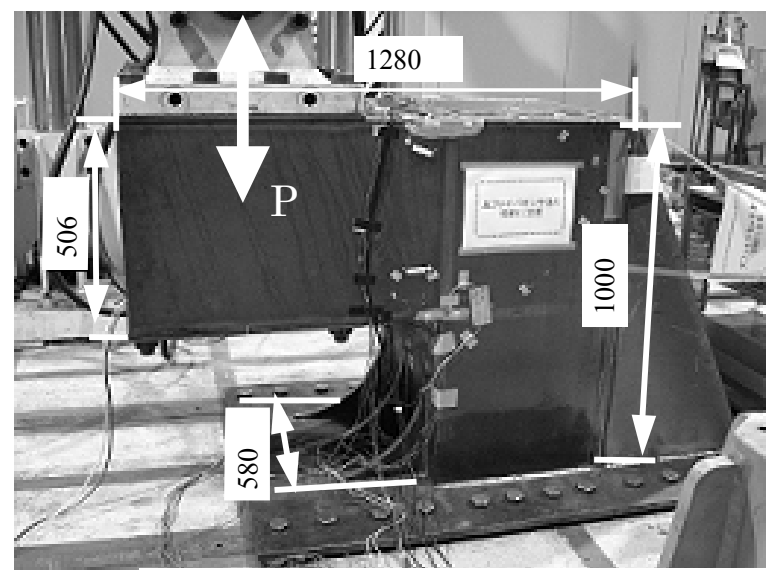

unit:mm

material:SS400

Fig.12 Image of test structure simulated L-shaped bridge foot 


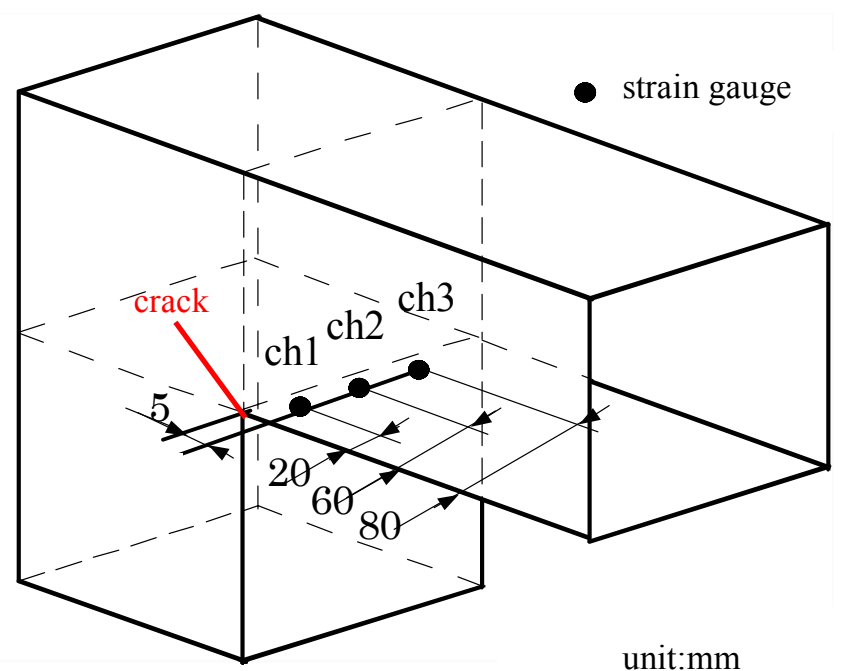

Fig.13 Schematic of set strain gauges and location of crack

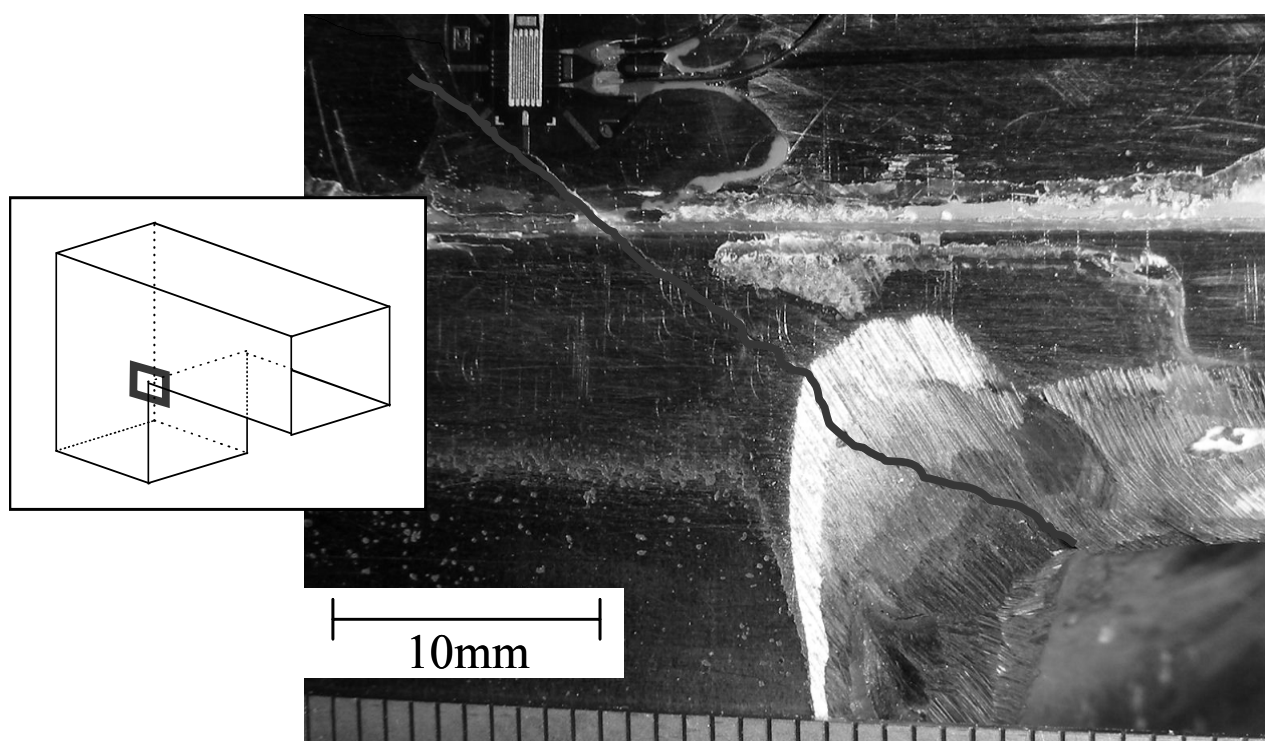

Fig.14 Image of growth of fatigue crack

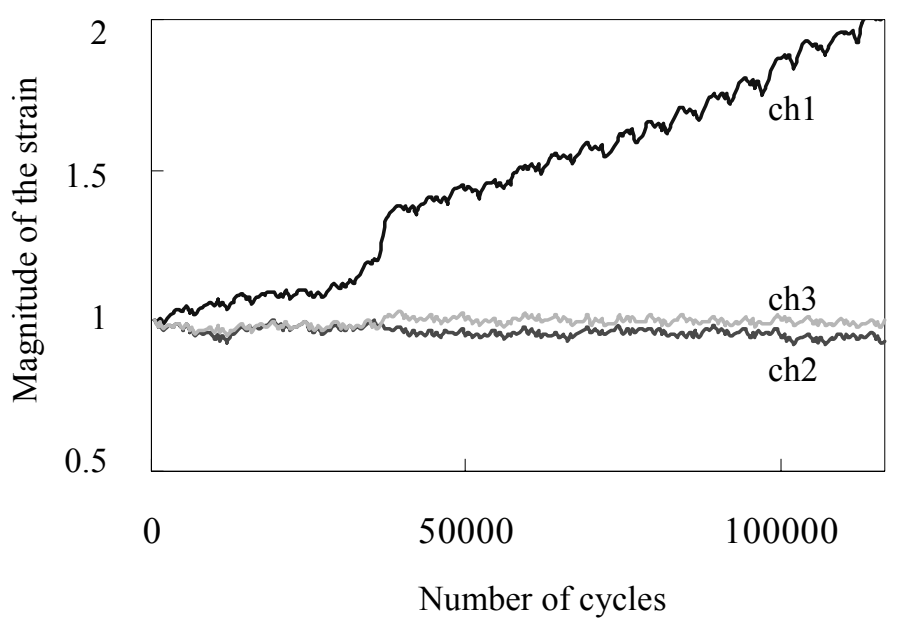

Fig.15 Magnitudes of normalized standard value of strain obtained from three strain gauges at each cycle 


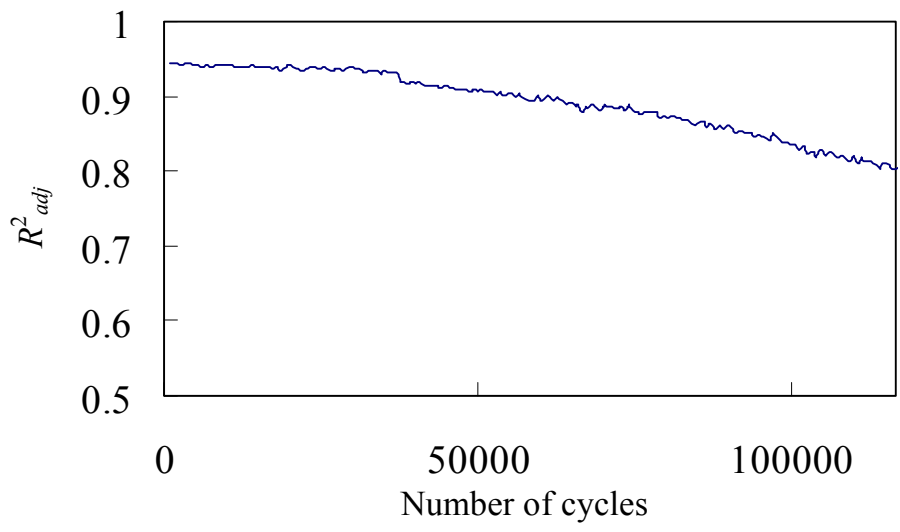

Fig. $16 R_{\text {adj }}^{2}$ at each cycle

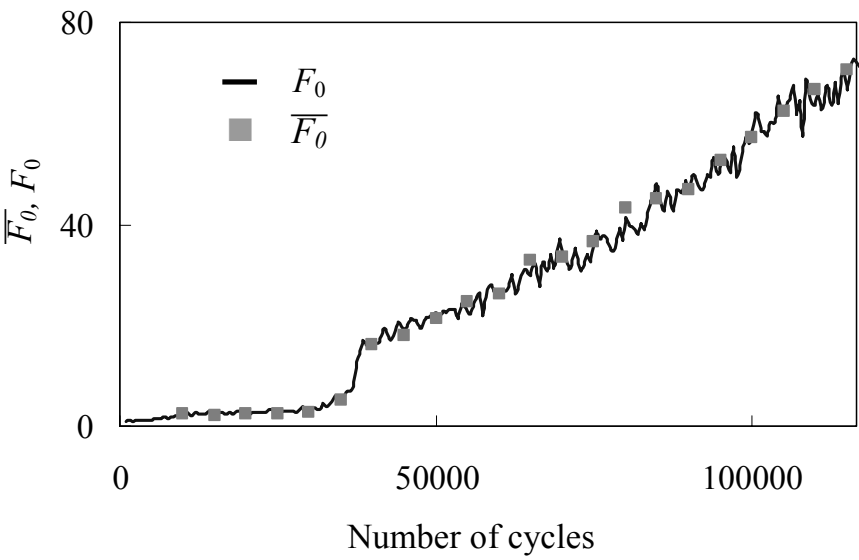

Fig. $17 F_{0}$, calculated by experimental data, and $\overline{F_{0}}$

\section{Conclusions}

Present study evaluates the effect of the accuracy of the regression of the response surface for the quantification of the relationship between the damage and the damage index.

The following results are obtained:

- Even if the change of correlation is small, $F_{0}$ increases high sensitivity as $R_{a d j}^{2}$ increases.

- As increase of fluctuation of sensor output by the damage of structure, $F_{0}$ increases high sensitivity even if low $R_{a d j}^{2}$.

- Although the sensitivity decreases by the change of correlation between the each explaining variable, changing the combination of each variables enables higher sensitivity diagnosis.

- $F_{0}$ varies only with the noise magnitude but is not affected by external forces

- The availability of the simulation is confirmed from the experiment.

\section{Acknowledgement}

This study was subsidized by JKA through its Promotion funds from KEIRIN RACE and was supported by the Mechanical Social Systems Foundation in Japan. The authors wish to acknowledge their appreciation.

\section{References}

1. Iwasaki, A., Todoroki, A., SHimamura, Y., Kobayashi, H., "Unsupervised Structural Damage Diagnostic Method Using Judgement of Change of Response Surface by Statistical Tool", Transactions of the Japan Society of Mechanical 
Engineers, Series A, Vol.68,No.673 (2002) , pp.1292-1297.

2. Iwasaki, A., Todoroki, A., SHimamura, Y., Sugiya, T., Izumu, S., Sakai, S., "Damage and Fault Diagnosis of In-service Structure via Statistical Comparison of Relation between Sensor measurements", Transactions of the Japan Society of Mechanical Engineers, Series A, Vol.72,No.724 (2006) , pp.1955-1962. 